International Journal of Pure and Applied Mathematics

Volume 84 No. 5 2013, 539-547

ISSN: 1311-8080 (printed version); ISSN: 1314-3395 (on-line version)

url: http://www.ijpam.eu

doi: http://dx.doi.org/10.12732/ijpam.v84i5.7

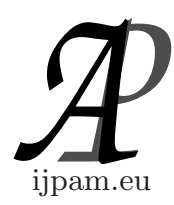

\title{
VOGAN DIAGRAMS OF AFFINE TWISTED LIE SUPERALGEBRAS
}

\author{
Biswajit Ransingh \\ Department of Mathematics \\ National Institute of Technology \\ Rourkela, INDIA
}

\begin{abstract}
A Vogan diagram is a Dynkin diagram with a Cartan involution of twisted affine superlagebras based on maximally compact Cartan subalgebras. This article construct the Vogan diagrams of twisted affine superalgebras.
\end{abstract}

AMS Subject Classification: 17B20, 17B22, 17B40

Key Words: Lie superalgebras, Vogan diagrams

\section{Introduction}

The real form of Lie superalgebra have a wider application not only in mathematics but also in theoretical physics. Classification of real form is always an important aspect of Lie superalgebras. There are two methods to classify the real form one is Satake or Tits-Satake diagram other one is Vogan diagrams. The former is based on the technique of maximally non compact Cartan subalgebras and later is based on maximally compact Cartan subalgebras. The Vogan diagram first introduced by A W Knapp to classifies the real form of semisimple Lie algebras and it is named after David Vogan. Since then the classification of Vogan diagram by different authors for affine Kac-Moody algebras (untwisted and twised), hyperbolic Kac-Moody algebras, Lie superalgebras and affine un-

Received: November 26, 2012

(c) 2013 Academic Publications, Ltd. url: www.acadpubl.eu 
twisted Lie superalgebras already developed. In this article we will developed Vogan diagrams of the rest superalgebras, twisted affine Lie superalgebras.

\subsection{The General Linear Lie Superalgebras}

Let $V=V_{\overline{0}} \oplus V_{\overline{1}}$ be a vector superspace, so that $\operatorname{End}(V)$ is an associative superalgebra. The $\operatorname{End}(V)$ with the supercummutator forms a Lie superalgebra, called the general linear Lie superalgebra and is denoted by $\mathfrak{g l}(m \mid n)$, where $V=\mathbb{C}^{m \mid n}$. With respect to an suitable ordered basis $\operatorname{End}(V)$ and $\mathfrak{g l}(m \mid n)$ can be realized as $(m+n) \times(m+n)$ complex matrices of the block form.

$\left(\begin{array}{ll}a & b \\ c & d\end{array}\right)$ where $a, b, c$ and $d$ are respectivily $m \times m, m \times n, n \times m$ and $n \times n$ matrices. The even subalgebra of $\mathfrak{g l}(m \mid n)$ is $\mathfrak{g l}(m) \oplus \mathfrak{g l}(n)$, which consists of matrices of the form $\left(\begin{array}{ll}a & 0 \\ 0 & d\end{array}\right)$, While the odd subspace consists of $\left(\begin{array}{ll}0 & b \\ c & 0\end{array}\right)$

Definition 1. A Lie superalgebras $\mathcal{G}$ is an algebra graded over $\mathbb{Z}_{2}$, i.e., $\mathcal{G}$ is a direct sum of vector spaces $\mathcal{G}=\mathcal{G}_{\overline{0}} \oplus \mathcal{G}_{\overline{1}}$, and such that the bracket satisfies

1. $\left[\mathcal{G}_{i}, \mathcal{G}_{j}\right] \subset \mathcal{G}_{i+j(\bmod 2)}$,

2. $[x, y]=-(-1)^{|x||y|}[y, x]$, (Skew supersymmetry) $\forall$ homogenous $x, y, z \in \mathcal{G}$ (Super Jacobi identity)

3. $[x,[y, z]]=[[x, y], z]+(-1)^{|x||y|}[y,[x, z]] \forall z \in \mathcal{G}$

A bilinear form $(.,):. \mathcal{G} \times \mathcal{G} \rightarrow \mathbb{C}$ on a Lie superalgebra is called invariant if $([x, y], z)=(x,[y, z])$, for all $x, y, z \in \mathcal{G}$

The Lie superalgebra $\mathcal{G}$ has a root space decomposition with respect to $\mathfrak{h}$

$$
\mathcal{G}=\mathfrak{h} \oplus \bigoplus_{\alpha \in \triangle} \mathcal{G}_{\alpha}
$$

A root $\alpha$ is even if $\mathcal{G}_{\alpha} \subset \mathcal{G}_{\overline{0}}$ and it is odd if $\mathcal{G}_{\alpha} \subset \mathcal{G}_{\overline{1}}$

A Cartan subalgebra $\mathfrak{h}$ of diagonal matrices of $\mathcal{G}$ is defined to be a Cartan subalgebra of the even subalgebra $\mathcal{G}_{\overline{0}}$. Since every inner automorphism of $\mathcal{G}_{\overline{0}}$ extends to one of Lie superalgebra $\mathfrak{g}$ and Cartan subalgebras of $\mathcal{G}_{\overline{0}}$ are conjugate under inner automorphisms. So the Cartan subalgebras of $\mathcal{G}$ are conjugate under inner automorphism. 


\section{Realization of Twisted Affine Lie Superalgebras}

Let $\mathcal{G}$ be a basic simple Lie superalgebra with non degenerate invariant bilinear form (.,.) and $\sigma$ an automorphism of finite order $m>1$. The eigenvalues of $\sigma$ are of the form $e^{\frac{2 \pi k i}{m}}, k \in \mathbb{Z}_{m}$ and hence admits the following $\mathbb{Z}_{m}$ grading:

$$
\mathcal{G}=\bigoplus_{k=0}^{m-1} \mathcal{G}_{k}, m \geq 2
$$

such that

$$
\left[\mathcal{G}_{i}, \mathcal{G}_{j}\right] \subset \mathcal{G}_{i+j}, i+j=i+j(\bmod m)
$$

and

$$
\begin{gathered}
\mathcal{G}_{k}=\left(\mathcal{G}_{k}\right)_{\overline{0}} \oplus\left(\mathcal{G}_{k}\right)_{\overline{1}} \\
\mathcal{G}_{k}=\left\{x \in \mathcal{G} \mid \sigma(x)=e^{\frac{2 \pi k i}{m} \cdot x}\right\}
\end{gathered}
$$

The twisted affine Lie superalgebra is defined to be

$$
\mathcal{G}^{(2)}=\left(\bigoplus_{k \in \mathbb{Z}_{m}} \mathbb{C} t^{k} \otimes \mathcal{G}_{k(\bmod m)} \oplus \mathbb{C} \mathbb{K} \oplus \mathbb{C} D\right.
$$

The Lie superalgebra structure on $\mathcal{G}^{2}$ is such that $c$ is the canonical central element and

$$
\begin{aligned}
{\left[x \otimes t^{m}+\lambda d, y\right.} & \left.\otimes t^{n}+\lambda_{1} d\right] \\
= & \left([x, y] \otimes t^{m+n}+\lambda n y \otimes t^{n}-\lambda_{1} m x \otimes t^{m}+m \delta_{m,-n}(x, y) c\right.
\end{aligned}
$$

where $x, y \in \mathcal{G}^{2}$ and $\lambda, \lambda_{1} \in \mathbb{C}$. The element $d$ acts diagonally on $\mathcal{G}$ with interger eigenvalues and induces $\mathbb{Z}$ gradation.

\subsection{Cartan Involution}

Let $\mathfrak{g}$ is a compact Lie algebra if the group Intg is compact. An involution $\theta$ of a real semisimple Lie algebra $\mathfrak{g}_{0}$ such that symmetric bilinear form

$$
B_{\theta}(X, Y)=-B(X, \theta Y)
$$

is positive definite is called a Cartan involution. 


\subsubsection{Cartan Involution of Contragradient Lie Superalgebras}

$B$ is the supersymmeytic nondegenerate invariant bilinear form on $\mathcal{G}$ define

$$
B_{\theta}(X, Y)=B(X, \theta Y)
$$

We say that a real form of $\mathcal{G}$ has Cartan automorphism $\theta \in \operatorname{aut}_{2,4}(\mathcal{G})$ if $B$ restricts to the Killing form on $\mathcal{G}_{0}$ and $B_{\theta}$ is symmetric negative definite on $\mathcal{G}^{(2)}$.

The bilinear form (., .) on $\mathcal{G}$ gives rise to a nondegenerate symmetric invariant form on $\mathcal{G}^{(m)}$ by

$$
\begin{gathered}
B^{(m)}\left(\mathbb{C}\left[t, t^{-1}\right] \otimes \mathcal{G}, \mathbb{C} K \oplus \mathbb{C} d\right)=0 \\
\Longrightarrow B^{(m)}\left(\bigoplus_{j \in \mathbb{Z}} t^{j} \otimes \mathcal{G}(\sigma)_{j \text { modm }}, \mathbb{C} K \oplus \mathbb{C} d\right)=0 \\
B^{(m)}\left(t^{j} \otimes X, t^{k} \otimes Y\right)=\lambda \delta^{j+k, 0} B(X, Y) \\
B^{(m)}\left(t^{j} \otimes X, K\right)=B^{(m)}\left(t^{j} \otimes X, d\right)=B^{(m)}(K, K)=B^{(m)}(d, d)=0 \\
B^{m}(c, d)=1
\end{gathered}
$$

Proposition 2. Let $\theta \in$ aut $_{2,4}\left(\mathcal{G}^{(m)}\right)$. There exists a real form $\mathcal{G}_{\mathbb{R}}^{(m)}$ such that $\theta$ restricts to a Cartan automorphism on $\mathcal{G}_{\mathbb{R}}^{(m)}$.

Proof. Since $\theta$ is an $\mathcal{G}^{(m)}$ automorphism, it preserves $B$. namely

$$
\begin{gathered}
B^{(m)}(X, Y)=B^{(m)}(\theta X, \theta Y) \\
B_{\theta}^{(m)}(X, Y)=B_{\theta}^{(m)}(Y, X), B_{\theta}^{(m)}(X, \theta X)=0 \\
B_{\theta}^{(m)}\left(X \otimes t^{m}, Y \otimes t^{n}\right)=B_{\theta}^{(m)}\left(Y \otimes t^{n}, X \otimes t^{m}\right)= \\
=t^{m+n} B(X, Y)
\end{gathered}
$$

for all $X, Y \in \mathcal{G}_{0}$

$$
B^{(m)}\left(K, X \otimes t^{k}\right)=B\left(D, X \otimes t^{k}\right)=B^{(m)}(D, D)=B^{(m)}(K, K)=0
$$

For $z \in L\left(t, t^{-1}\right) \otimes \mathcal{G}_{0}$ and $X, Y \in L\left(t, t^{-1}\right) \otimes \mathcal{G}_{1}$

$$
B_{\theta}^{(m)}(X,[Z, Y])=B^{(m)}(X,[\theta Z, \theta Y])=-B_{\theta}^{(m)}(X,[\theta Z, \theta Y])
$$




$$
B_{\theta}^{(m)}(X,[Z, Y])=0
$$

$\forall X \in \mathbb{C} K$ or $\mathbb{C} D$

$\mathcal{G}_{\mathbb{R}}^{(m)} \simeq \mathcal{G}_{\overline{0} \mathbb{R}}^{(m)} \simeq \mathcal{G}_{\overline{0} \mathbb{R}}$. The above three real forms are isomorphic. So the Cartan decomposition of $\mathcal{G}_{\mathbb{R}}^{(m)}$ are isomorphic

to $\mathcal{G}_{\overline{0}}$.

$\mathcal{G}_{\overline{0}}=\mathfrak{k}_{0} \oplus \mathfrak{p}_{0}$

$B_{\theta}(X,[Z, Y])=\left\{\begin{array}{cc}-B_{\theta}([Z, X], Y) & \text { if } Z \in \mathfrak{k}_{0} \\ B_{\theta}([Z, X], Y) & \text { if } Z \in \mathfrak{p}_{0}\end{array}\right.$

We say that a real form of $\mathcal{G}$ has Cartan automorphism $\theta \in \operatorname{aut}_{2,4}(\mathcal{G})$ if $B$ restricts to the Killing form on $\mathcal{G}_{0}$ and $B_{\theta}$ is symmetric negative definite on $\mathcal{G}_{\mathbb{R}} . B_{\theta}\left(X_{i}, X_{j}\right)=\delta_{i j}$. It follows that $B_{\theta}$ negative definite on $\mathcal{G}_{\overline{1} \mathbb{R}}^{(m)}$. By $B_{\theta}$ is symmetric bilinear form on $L_{1}\left\{1 \otimes X_{1}, 1 \otimes X_{2}, \cdots, d\right\}$. So it is conclude that $\theta$ is a Cartan automorphism on $\mathcal{G}^{(m)}$.

\section{Vogan Diagram}

Let $\mathfrak{g}_{0}$ be a real semisimple Lie algebra, Let $\mathfrak{g}$ be its complexification, let $\theta$ be a Cartan involution, let $\mathfrak{g}_{0}=\mathfrak{k}_{0} \oplus \mathfrak{p}_{0}$ be the corresponding Cartan decomposition A maximally compact $\theta$ stable Cartan subalgebra $\mathfrak{h}_{0}=\mathfrak{k}_{0} \oplus \mathfrak{p}_{0}$ of $\mathfrak{g}_{0}$ with complexification $\mathfrak{h}=\mathfrak{k} \oplus \mathfrak{p}$ and we let $\triangle=\triangle(\mathfrak{g}, \mathfrak{h})$ be the set of roots. Choose a positive system $\triangle^{+}$for $\triangle$ that takes $i \mathfrak{t}_{0}$ before $\mathfrak{a} . \theta\left(\triangle^{+}\right)=\Delta^{+}$

$\theta\left(\mathfrak{h}_{0}\right)=\mathfrak{k}_{0} \oplus(-1) \mathfrak{p}_{0}$. Therefore $\theta$ permutes the simple roots. It must fix the simple roots that are imaginary and permute in 2-cycles the simple roots that are complex. By the Vogan diagram of the triple $\left.\left(\mathfrak{g}_{0}, \mathfrak{h}_{0}, \Delta^{+}\right)\right)$., we mean the Dynkin diagram of $\Delta^{+}$with the 2 element orbits under $\theta$ so labeled and with the 1-element orbits painted or not, according as the corresponding imaginary simple root is noncompact or compact.

\section{Twisted Affine Lie Superalgebras}

A Dynkin diagram of $\mathcal{G}^{(m)}$ is obtained by adding to the Dynkin diagram of $\mathcal{G}$.

\subsection{Root Systems}

$$
O S p(2 m \mid 2 n)^{(2)}
$$




$$
\triangle=\left\{\frac{k}{2}-\delta_{1}, \delta_{1}-\delta_{2}, \cdots, \delta_{n-1}-\delta_{n}, \delta_{n}-e_{1}, e_{1}-e_{2}, \cdots, e_{m-1}-e_{m}, e_{m}\right\}
$$

The $\mathcal{G}_{0}$ representation $\mathcal{G}_{1}$ is the fundamental representation of $O s p(2 m-1 \mid 2 n)$ whose lowest weight is $-\delta_{1}$.

$$
\operatorname{OSp}(2 \mid 2 n)^{(2)}
$$

There exist an automorphism $\tau$ such that the invariant subsuperalgebra $\mathcal{G}_{0}$ is $O S p(1 \mid 2 n)$. The $\mathcal{G}_{0}$ The simple root system of $\mathcal{G}_{0}$ is

$$
\triangle=\left\{\delta_{1}-\delta_{2}, \cdots, \delta_{n-1}-\delta_{n}, \delta_{n}\right\}
$$

The lowest weight of the $\mathcal{G}_{1}$ representation of $\mathcal{G}_{0}$ is $\delta_{1}$.

$$
\operatorname{Sl}(1 \mid 2 n+1)^{(4)}
$$

The invariant subalgebra can be taken to as $O(2 n+1)$ and the lowest weight is $-\delta_{1}$

\section{Vogan Diagrams of Affine Lie Superalgebras}

Let $c$ the circling of vertices, $d$ diagram involution, $a_{s}$ numerical labeling and $D$ Dynkin diagram of $\mathcal{G}^{(m)}$. Note- $S$ is defined in the next proposition.

Definition 3. A Vogan diagram $(c, d)$ on $D$ and one of the following holds:

- $\theta$ fixes grey vertices

- $\theta$ interchange grey vertices and $\sum_{S} a_{\alpha}$ is odd.

- $\sum_{S} a_{\alpha}$ is odd

The $\theta, \delta$ and $c$ are expressed in terms of the bases given as follows

$$
\theta=\sum_{i=1}^{n} a_{i} \alpha_{i}, \delta=\sum_{i=0}^{n} a_{i} \alpha_{i}
$$

Fix a set $\pi$ of simple roots of $\mathcal{G}$, we take $\hat{\pi}=\left\{\alpha_{0}=\delta-\theta\right\} \cup \pi$ be the simple roots of $\hat{\mathcal{G}}^{(m)}$. $\theta$ is the highest weight in $\triangle_{0}^{(1)} \cup \triangle_{1}^{(1)}$.

If $\theta$ extend to aut $_{2,4}$, (automorphism of order 2 or 4 ) then $\theta$ permutes the extreme weight spaces $\mathcal{G}^{(m)}$. Since $\theta \mid \mathcal{G}_{0}$ is represented by $(c, d)$ on $D_{0}$, it permutes the simple root spaces of $\mathcal{G}_{0}$. Hence $\theta$ permutes the lowest weight spaces of $\mathcal{G}^{(m)}$ and d extend to $\operatorname{inv}\left(X^{(m)}(m, n)\right)$ 
Proposition 4. Let $\mathcal{G}_{\mathbb{R}}$ be a real form, with Cartan involution $\theta \in \operatorname{inv}\left(\mathcal{G}_{\mathbb{R}}\right)$ and Vogan diagram $(c, d)$ of $D_{0}$. The following are equivalent

(i) $\theta$ extend to aut $_{2,4} \mathcal{G}^{(m)}$.

(ii) $\left(\mathcal{G}_{\overline{0} \mathbb{R}}\right)$ extend to a real form of $\mathcal{G}^{(m)}$.

(iii) $(c, d)$ extend to a Vogan diagram on $D$

Proof. Let $S$ be the $d$ - orbits of vertices defined by [4]

$$
S=
$$

\{vertices painted by $\mathrm{p}\}$

\{white and adjacent 2-element d-orbits\}

\{grey and non adjacent 2-element d-orbits\}

Let $D$ be the Dynkin diagram of $\left.\mathcal{G}^{(m)}\right)$ of simple root system $\Phi \cup \phi(\Phi$ simple root system with $\phi$ lowest root) with $D=D_{\overline{0}}+D_{\overline{1}}$, where $D_{\overline{0}}$ and $D_{\overline{1}}$ are respectively the white and grey vertices. The numerical label of the diagram shows $\sum_{\alpha \in D_{\overline{1}}}=2$ has either two grey vertices with label 1 or one grey vertex with label 2.

(i) $D_{\overline{1}}=\{\gamma, \delta\}$ so the labelling of the odd vertices are 1 .

(ii) $D_{\overline{1}}=\{\gamma\}$ so labelling is $2\left(a_{\alpha}=2\right)$ on odd vertex.

$\theta \in \operatorname{inv}\left(\mathcal{G}_{\mathbb{R}}\right) ; \theta$ permutes the weightspaces $L\left(t, t^{-1}\right) \otimes \mathcal{G}_{\overline{1}}$ The rest part of proof of the proposition is followed the proof of the propostion 2.2 of [3]

Sum of the $a_{s}$ of odd root is 2. When there is a $\sigma$ stable compact Cartan subalgebra is $\mathfrak{h} \oplus C c \oplus D d$ then the Vogan diagrams are the following.

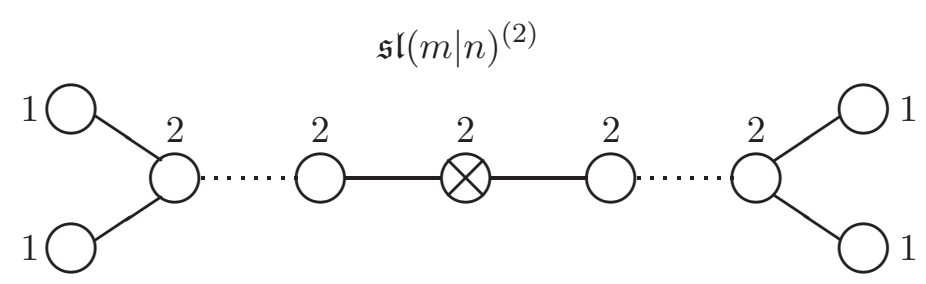



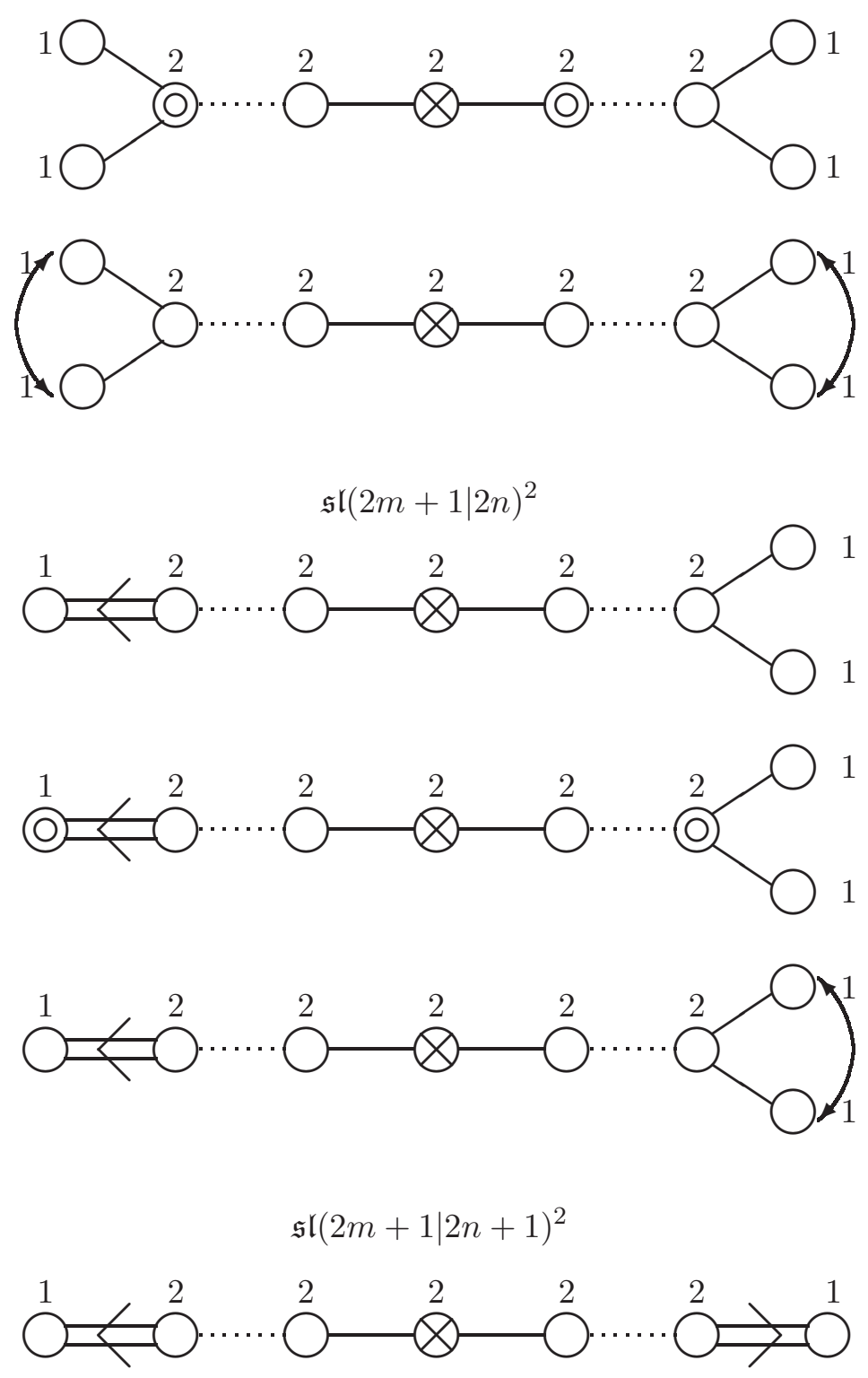

$\mathfrak{s l}(2 \mid 2 n)^{2}$ 


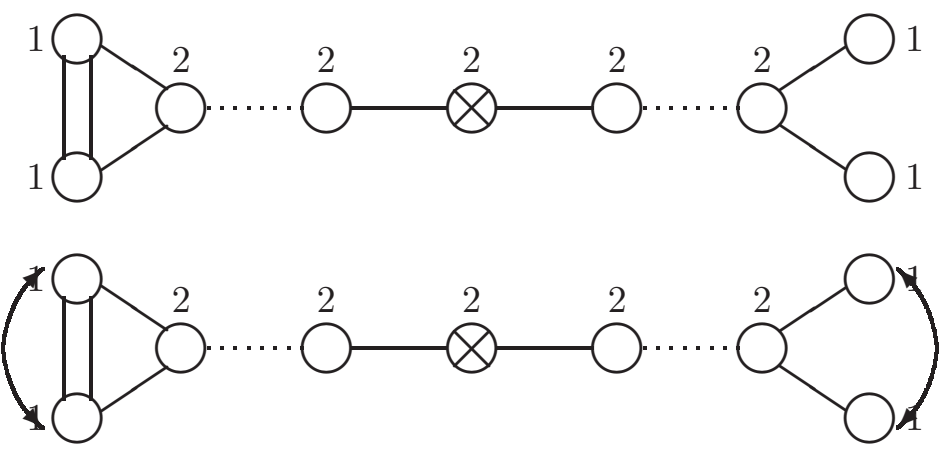

References

[1] P. Batra, Vogan diagrams of affine Kac-Moody algebras, Journal of Algebra, 251 (2002), 80-97.

[2] P. Batra, Invariant of real forms of affine Kac-Moody Lie algebras, Journal of Algebra, 223 (2000), 208-236.

[3] Chuah Meng-Kiat, Cartan automorphisms and Vogan superdiagrams, Mathematische Zeitschrift (2012).

[4] Chuah Meng-Kiat, Finite order automorphism on contragredient Lie superalgebras, Journal of Algebra, 351 (2012), 138-159.

[5] L. Frappat, A. Sciarrino, Sorba, Structure of basic Lie superalgebras and of their affine extensions, commun. Math. Phys., 121 (1989), 457-500.

[6] V.G. Kac, Infinite Dimensional Lie Algebras, Birkhuser, USA (2003).

[7] V.G. Kac, Lie superalgebras, Adv. Math., 26 (1977), 8-96.

[8] A.W. Knapp, Lie Groups beyond an Introduction, Birkhuser, Boston (2002).

[9] M. Parker, Classification of real simple Lie superalgebras of classical type, J. Math. Phys., 21, No. 4 (1980), 689-697.

[10] B. Ransingh, K.C. Pati, Vogan diagrams of basic Lie superalgebra, ArXiv: 1205.1394v1 [math.RT] (2012). 
